\title{
Magnetotelluric imaging of basalt-covered sediments
}

\author{
D. Beamish ${ }^{1}$ and J.M. Travassos ${ }^{2,3}$ \\ ${ }^{1}$ British Geological Survey, Keyworth, Nottingham NG12 5GG, UK. ${ }^{2}$ Departmento de Geophysica, CNPQ Observatorio Nacional, R. \\ Gen. Bruce 586, 20291 Rio de Janeiro, Brazil. \\ ${ }^{3}$ Also at: PETROBRAS/CENPES/SEGEF, 21910 Rio de Janeiro, Brazil.
}

Beamish, D. and Travassos, J.M., 1992. Magnetotelluric Imaging of basalt-covered

sediments. First Break, 10, 345-357. DOI: 10.3997/1365-2397.1992018

\section{Introduction}

The magnetotelluric (MT) technique has evolved as a tool in difficult exploration problems such as seismic poor record areas (Orange 1989) and in frontier basin regions (Christopherson 1991). Since it is a deep-probing wavefield technique, MT can assist in confirming the presence of deep sedimentary rocks and in providing estimates of the thickness of surface formations and the broad structural features of both sediments and basement.

The intracratonic Parana basin of South America presents one such difficult, and large, exploration problem. The major part of the basin, some $800000 \mathrm{~km}^{2}$, is covered with basalts up to $2 \mathrm{~km}$ thick (Fig. 1), making this the world's most voluminous flood basalt complex. The basalts overlie a series of Palaeozoic sediments containing known hydrocarbon source and reservoir rocks (Milani et al. 1990). The early 1980s saw a very active phase of exploration within the Brazilian sector of the basin when numerous seismic lines were acquired along with control wells and magnetotelluric profiles.

Stanley et al. (1985) have previously discussed the application of regional MT surveys in relation to hydrocarbon exploration within the basin. This study considers a more detailed grid survey with site separations of $2 \mathrm{~km}$ undertaken in the Clevelandia area (Fig. 1). The survey area contains both seismic reflection lines and an exploration well, logged through to basement (3850 m). The MT data, being over ten years old, possess a number of limitations which are discussed herein. However, despite these limitations the data characteristics indicate that an assessment of the vertical structure across the basin is a valid exercise. The data are therefore used in a limited way to assess only the vertical resolution capabilities of the MT method when applied to this type of exploration problem. We are fully aware that a modern survey would provide better data together with a greater control of statics and therefore offer a more rigorous interpretation.

The seismic sections from the Clevelandia survey are of poor quality and have not been interpreted. As a consequence, the main control on the MT interpretation lies in the information from the survey well. The resistivity induction log is discussed in some detail and it is emphasized that the incorporation of the available information within the MT interpretation is a complicated exercise. The difficulties arise due to the low-order vertical resolution of the MT wavefield. This can result in a complicated relationship between the geoelectric units resolved by surface soundings and information obtained at a different scale. 
The data detect three main units before electrical basement. The resistivity and thickness $(1200 \mathrm{~m})$ of the mid-Early Cretaceous flood basalts are both well resolved. The complete Palaeozoic sedimentary sequence is represented by two units, the lower being expressed as a highly conducting unit (of order $1 \mathrm{ohm} . \mathrm{m}$ ) in the vicinity of formations which contain hydrocarbon source shales.

Resistivity sections across the basin are constructed using both layered and smooth (non-interface) inversion results. The smooth vertical profiles are less susceptible to parameter equivalencing since they possess minimum structure and appear more stable when cross-sections are constructed. Cross-sections obtained using minimum structure resistivity models are defined in terms of a set of gradients and turning points. In order to enhance the more conventional interface-type attributes of such data, further processing has been applied. Using the control provided by the well log, the application of a simple shadow relief technique and a more formal reflectivity transformation are demonstrated.

\section{The MT data}

The MT data discussed here were obtained by a commercial contractor in the early 198Qs. The data have a number of limitations which are discussed below. Our main purpose is to re-examine these data using some new procedures with a view to understanding the capabilities of MT when applied to this type of exploration problem.

The complete grid survey, undertaken at about $2 \mathrm{~km}$ centers, is shown in Fig. 2. The location of well 2-AL-1- SC is also indicated. The MT data were acquired in conjunction with seismic reflection lines crossing the Clevelandia survey area. The quality of these data is poor and they have not been interpreted. In our view the time-migrated sections that are available would add virtually no valid control in relation to the models developed from the MT data.

The available sounding data comprise only the main off-diagonal tensor elements ( $\rho x y, \phi x y$ and $\rho y x$, $\phi y x)$ in the measured $(x, y)$ coordinates. This means that we cannot apply formal tensor decomposition techniques to determine the two-dimensional and/or three-dimensional contributions to our data. In our opinion this is not a serious limitation in that the data characteristics suggest a high degree of one-dimensional (1D) behaviour across the high-frequency portion of the bandwidth, down to about $0.1 \mathrm{~Hz}$. A second limitation of the data is that no additional survey information is available with regard to the control of statics. Static distortion effects (e.g. Jiracek 1990) provide parallel offsets in the apparent resistivity data (the amplitude information) but leave the phase data (containing the time terms) undistorted. Such effects are pervasive throughout the Clevelandia survey data.

The scheme adopted to correct the data for static distortion is described by Beamish and Travassos (1992). Briefly, the method involves the use of parametric constraints, applied as uniform layer resistivities, together with phase-only data inversion. These procedures provide estimates of the frequency-independent gain factors for both components which are then used to correct the measured apparent resistivity data. We use the data from the east-west profile formed by 'line 5' (Fig. 2) to provide examples of the cross-sectional behavior of the data. This line contains two soundings (sites 8 and 10) near the control well.

The corrected sounding data, in both the $x y$ and yx components, from 14 sites along this profile are shown in Fig. 3. The data characteristics observed are consistent with a conceptual model of a large- 
scale sedimentary basin in which a set of 1D models is valid down to a low-frequency limit.

Anisotropy between the components starts to become significant for frequencies below about 0.1

$\mathrm{Hz}$ which corresponds to penetration depths of 3000-4000 m. The data suggest that a 1D assumption will only introduce significant errors in the basement structure. In keeping with the conceptual model and the data characteristics (Fig. 3) we use the Berdichevsky invariant formed from the two components (half the difference of the off-diagonal impedance elements) to undertake assessments of the vertical structure of the basin.

\section{The well-log control}

The deep induction log (1 m sampling) from well 2-AL- 1-SC is shown in Fig. 4. The log runs from 100 $\mathrm{m}$ to $3881 \mathrm{~m}$. In principle, the log should measure the true resistivity of the uninvaded zone well beyond the borehole. Formation stratigraphy is indicated by the horizontal dotted lines. The base of the Serra Geral flood basalt (a) is the first stratigraphic marker at $1230 \mathrm{~m}$ and beneath this horizon a sequence of -marine and continental Palaeozoic sedimentary units occur (Zalan et al. 1986). The Triassic-Jurassic Botucatu Formation (b) comprises mainly sandstones. The upper Permian Rio do Rasto Formation (c) consists of brick-red sandstones and some shales and silts. The Terezina, Serra Alta and Irati Formations ( $d, e, f)$ consist largely of shales with some minor siltstone. The Palermo and Rio Bonito Formations ( $g, h$ ) comprise largely siltstone and sandstone. The Harare Formation (i) consists of shale, diamictite, argillite, some sandstone and a few thin coal beds. The well encounters Silurian sediments within the Furnas Formation (j) and enters metagabroic basement at $3850 \mathrm{~m}$. A number of thin diabase intrusives occur throughout the sedimentary sequence and their locations are indicated by the letter $D$ in Fig. 4. In terms of basin-wide hydrocarbon potential, the Permian shale sequence within the Irati Formation $(f)$ is considered to be a possible source rock (Milani et al. 1990). In the case of 2-AL-1-SC, the formation is intruded by a sill whose thermal impact will have influenced hydrocarbon maturation.

The progressive conductance (inverse resistivity integrated with depth) is also shown in Fig. 4. The main changes in gradient of this parameter can be used to define equivalent uniform layers (Keller 1987). Both the resistivity and conductance profiles of Fig. 4 provide a good example of the wavenumber content of a deep resistivity well-log. The largest variations occur at the scale of meters and relate to thin-bed lithology (including here intrusives) rather than formation stratigraphy per se. The only substantial transition in bulk (vertically averaged) resistivity occurs between the flood basalts and the underlying sediments. At greater depths a low-wave-number contribution, associated with the minimum in the resistivity profile, is also very evident. Both profiles shown in Fig. 4 indicate that there is very little correlation between bulk resistivity values (i.e. equivalent layers) and the stratigraphic column.

\section{MT resolution characteristics}

The situation regarding resolution when using the plane-wave MT wavefield is complicated by the fact that the downgoing-wavefield experiences dispersion. This simply means that, unlike the acoustic case, velocity is proportional to both a material property (in this case resistivity) and the frequency of the wavefield used. This means that when we come to examine the inverse scattering problem, depth and resistivity must be solved for simultaneously. The dispersion inherent in the method results in a relatively low resolution of vertical structure. The wavefield parameter most common in MT is 'skin-depth' which refers to a scale length across which a significant level of attenuation (in amplitude) occurs within a uniform medium. If we take a resistivity of $10 \mathrm{ohm} . \mathrm{m}$ to 
be representative of the sedimentary sequences (Fig. 4) and a frequency range of $2 \mathrm{SO} \mathrm{Hz}$ to 0.001 $\mathrm{Hz}$, as used here, then skin-depths would range from $100 \mathrm{~m}$ to $50 \mathrm{~km}$, respectively. It is also possible to estimate the phase velocity and wavelength of the down-going electric field. For this example, phase velocities would range from $158 \mathrm{~km} \cdot \mathrm{s}^{-1}(250 \mathrm{~Hz})$ to $0.32 \mathrm{~km} \cdot \mathrm{s}^{-1}(0.001 \mathrm{~Hz})$ and wavelengths would range from $632 \mathrm{~m}(250 \mathrm{~Hz})$ to $316 \mathrm{~km}(0.001 \mathrm{~Hz})$.

The wavelengths involved mean that the technique cannot intrinsically resolve thin-bed formations unless very high frequencies (and therefore shallow targets) are involved. Resolution decreases with depth and favours low-resistivity, thick formations. Simple forward modelling studies indicate that the existence of a single, buried layer can only be resolved if its conductance (conductivity-thickness product) exceeds that of the overlying formations.

\section{D layered inversions}

A large number of algorithms exist which solve the inverse scattering problem in terms of a 1D layered model using model parameters of thickness and resistivity. The different formulations all attempt to minimize the error between the observed data and that of a theoretical, minimum-norm model. One of the problems associated with layered model construction is that we need to know the 'correct' number of layers (or equivalently the number of 'reflectors' (Gibert and Virieux 1991)). This problem is usually investigated by performing multiple data inversions from an initial half-space model which comprises successively increasing numbers of layers. The initial model, with the minimum number of layers, which gives a satisfactory misfit is then identified as a correct choice for final model construction. The layered inversion scheme used here is that due to Fischer and Le Quang (1981).

As an example, Fig. 5a shows two model constructions, using 4- and 7-layers, at sites 8 (left) and 10 (right). Only the first $4 \mathrm{~km}$ of the solutions are shown. The 7-layer model is shaded in each case and the heavy dash line indicates the base of the basalt at the nearby well. Fig. $5 b$ shows the responses of these two models at site 8 in relation to the observed data. The 4-layer model achieves an r.m.s. misfit of 1.16, close to the expectation level of the data (unity). The 7-layer model provides a much lower misfit of 0.51 and so considerably overfits the data.

The 4-layer model indicates a uniform basalt followed by two uniform geoelectric units within the main sedimentary section prior to basement. The 7-layer model achieves a close fit to the high frequency phase data (Fig. 5b) by incorporating an 'at-surface' conductive layer. This layer cannot be resolved since it is observed only asymptotically in the data. Apart from the at-surface layer both models agree that three main geoelectric units are resolved above basement. The interpretation difficulty is that the two forms of model give rise to different vertical profiles due to the problem of equivalences between model parameters.

Equivalence, or sensitivity analysis, is a straightforward part of the interpretation procedure (e.g. Pedersen and Hermance 1986). When applied to the present data it indicates that the resistivity and thickness of layer 1 (the basalt) are both highly resolved parameters but only the conductance (resistivity-thickness product) of the highly-conducting layer 3 is resolved. This behaviour is most evident in the variance displayed in the model parameters in Fig. 5.

At this point in the modelling exercise it would be valuable to increase the resolution of our models (decrease the parameter trade-offs) by incorporating one or more parameter constraints using the 
control provided by the induction log. A comparison of the unconstrained inversions of Fig. 5 and the induction log shown in Fig. 4 indicates at least two major difficulties. The first is that, although the resistivity and thickness of the basalt layer compare very well between well-log and models, the modelled resistivities of the most conducting layer appear to be significantly lower than those measured by the induction log. This 'offset at depth' applies to all our vertical profiles and is discussed later. The second difficulty is that at wavenumbers which are equivalent to the resolution of our data (exemplified by the layered models), it is by no means clear from the induction log that a layered interpretation space is appropriate. Apart from the transition in bulk (vertically-averaged) resistivity between the basalt cover and sediments the induction log appears to be well described by a number of reasonably smooth and low-order trends. It seems legitimate to suggest that, at wavenumbers appropriate to the resolution of MT data, gradational behaviour may be a more appropriate description of the in-situ resistivity variation.

\section{D smooth inversions}

Formulations of the inverse scattering problem when the vertical resistivity profile to be recovered is rigorously smooth (e.g. in terms of its first or second derivatives), have been considered by Constable et al. (1987) and by Smith and Booker (1988). A technique for smooth inversion which incorporates a priori information from well logs has been presented recently by Pilkington and Todoeschuck (1991). In view of the preceding discussion we consider this to be an important development. It can be argued that these regularized smooth models are desirable since they have minimal structure and, hence, features observed in them should have a greater likelihood of being representative of the true resistivity profile (Oldenburg 1990).

Such formulations dispense with the requirements that the numbers of 'layers' to be modelled should be known or established. To some extent a smooth solution should identify the number of 'equivalent' layers in terms of turning points and changes in gradient of the model returned. Figure 6 is a comparison of smooth inverse models obtained along profile 5 and their corresponding fourlayer counterparts. The smooth solutions were obtained using the OCCAM algorithm of Constable et al. (1987) at an r.m.s. misfit of 1.3. The smooth models incorporate 30 layers per decade in depth and the models are compared in the 'natural' depth scale for MT which is logarithmic.

The smooth models shown in Fig. 6 demonstrate a form of correspondence with their layered counterparts. The essential difference, apart from the lack of interfaces, is that the smooth solutions allow resistivity gradients to exist in what would otherwise be a fixed resistivity layer. The resistivity gradients that occur through the upper kilometer of these smooth models clearly indicate a nonuniform basalt resistivity in keeping with that of the induction log. The smooth models at sites 8 and 10, near the well, are compared with the induction log in a two-stage depth scale in Fig 7a and b. Within the first two kilometers the models provide gradients which appear to be a good representation of the behaviour of the induction log. The behaviour shown in Fig. 7a is an example of the way in which a resistivity transition, at the base of the basalt, is represented by a smooth model.

Following the local minimum at a depth of about $1400 \mathrm{~m}$, the model profiles display a slight increase through the uppermost sedimentary sequence before decreasing to a profile minimum at a depth of about $2700 \mathrm{~m}$. Below $2000 \mathrm{~m}$ (Fig. 7b) an offset develops between the induction log and the resistivity profiles returned by the inversion. The offset continues, although the gradients differ, with 
increasing resistivity through to basement. Since a good amplitude correspondence is obtained through the upper section, residual static distortion in the sounding data cannot be the cause. This effect occurs repeatedly in other regions of the Parana Basin (i.e. in comparisons between other well logs and associated MT data). The offset is gradational, increases with depth and is largest in the vicinity of the most conductive formations. In order to verify that the offset is not simply a consequence of inadequate (i.e. 1D) modelling, investigations into the behaviour of resistivity crosssections obtained by 2D inversion were carried out. Such investigations are necessarily restricted by the lack of full tensor information. In the circumstances, E-W profiles were considered and it was assumed that the xy component data related to a TM-mode (the electric field perpendicular to strike) and that the $y x$ component data then related to a TE-mode of induction (the electric field parallel to strike). Treated in this way the data examine pure E-W structural strike on the basis that this is either 'true' or 'more realistic than the ID assumption'. The alternative vice versa formulation which examines the assumption of pure N-S structural strike was also considered.

20 regularized (smooth) inversion (deGroot-Hedlin and Constable 1990) was undertaken along each of the six E-W profiles using eight evenly-spaced frequencies and using 20 layers per decade of depth in model construction. The starting point of each inversion was a half-space of $50 \mathrm{ohm} . \mathrm{m}$. In general, adequate total r.m.s. misfits (of order 2.5-3.5) were obtained by the OCCAM-2D inversion algorithm. The $2 \mathrm{D}$ inversions consistently returned minimum resistivities of several ohm.m at depths of the same order as those of Fig. 7b $(2700 \mathrm{~m})$. In summary we find that a very similar 'offset', in both form and magnitude, exists in both $1 \mathrm{D}$ and 2D treatments of the data. Due to the restrictive nature of the assumptions required to enable 2D modelling to be undertaken here, it is suggested that the 1D approach (using the geometric mean of horizontal resistivities) is probably the more cautious method of structural appraisal for the data here considered.

The comparisons of Figs 6 and 7 demonstrate some of the practical limitations of the MT technique when imaging a complex variation of in situ resistivity with depth. The modelled profiles display some correspondence with the main features of the stratigraphic column but not in a simple one-toone manner. In Fig. 7 the first maximum in the modelled profile marks the transition from an upper basalt sequence to a lower one of increased resistivity. We associate this transition with a change from slightly porphyritic acid flows in the upper section to mainly basic lavas in the lower section. The base of the flood basalts is associated with a local maximum in the gradient of the smooth model (see later). A local minimum then occurs in the vicinity of the second stratigraphic marker. Below $2000 \mathrm{~m}$ the model resistivity descends to a profile minimum in the vicinity of the top of the Itarare Formation. Below $2700 \mathrm{~m}$ the resistivity of the model profile increases in a manner similar to the induction log through to basement $(3850 \mathrm{~m})$.

Despite the rather broad resolution attributes of the MT models it is suggested that both the gradients and the turning points of the smooth solutions can provide a useful mapping capability away from the control provided by the well log. In order to demonstrate this capability, a resistivity cross-section (logarithmic resistivity scale) is shown in Fig. 8a. The section shown is contoured from the 14 smooth solutions across profile 5 . We emphasize that the result is a property cross-section and structural (e.g. stratigraphic) horizons can only be inferred using the well log control discussed above. The flood basalt cover is clearly of highly uniform thickness along the $28 \mathrm{~km}$ of the section. Across the eastern portion of the section the higher resistivities found within the upper sedimentary sequences may reflect a larger contribution from resistive sills (this is speculative). The minimum 
resistivity, associated with the top of the Itarare formation, is mapped as a conductive unit (resistivities of $<3$ ohm.m) horizontal in the east but with an implied sub-horizontal component of dip in the west. The slight variations that occur in the resistivities of the deeper part of the section should be treated with caution due to the limitations of the present 1D assessment.

There is an inevitable tendency to interpret a resistivity (property) cross-section in structural and/or stratigraphic terms. This can be hazardous since the contoured levels are often chosen for convenience rather than being based on a stratigraphic tie-in. It is, however, possible to exploit the properties of our smooth, minimum- structure models to obtain more structurally related crosssections.

\section{Further processing}

As discussed previously, the particular features that 'define' a smooth model are the gradients and turning points (e.g. Figs 6 and 7). It is therefore possible to visually enhance the variations in the vertical gradients obtained across a section using the same shadow-relief techniques that have been applied with much success to potential field maps. It should be noted that here our 'data' are the result of stable, regularized inversion and will contain only low wavenumber components.

Figure $8 \mathrm{~b}$ shows the resistivity cross-section of Fig. 8a processed using shadow-relief. The crosssection is illuminated at an elevation of $45^{\circ}$ and at an angle of $180^{\circ}$ (i.e. from the south or 'below'). When applied to a cross- section this angle preferentially illuminates strictly horizontal gradients. Starting at the surface and working down, the shadow-relief plot picks out the major gradients associated with the upper/lower flood basalt transition, the base of the flood basalts, gradients associated with the upper sedimentary sequence and finally the largest gradients associated with the minimum resistivities across the section. The low values of the resistivity gradients within the basement are satisfactorily represented by a nearly uniform grey tone. The illumination angle emphasizes possible sub-horizontal structural features between easting values of 71 and $83 \mathrm{~km}$.

Figure $8 \mathrm{~b}$ suggests that shadow-relief techniques can provide a useful mapping of the lateral correlation of the resistivity gradients associated with minimum-structure models. It is also possible to undertake a related, but more formal, analysis of such properties using the definition of reflection coefficients that arises due to the boundary conditions between different electrical media. The MT reflection coefficient $\left(r_{j}\right)$ between two layers of resistivities $\rho_{j}$ and $\rho_{j+1}$ is defined as:

$$
r_{j}=\frac{\sqrt{\rho_{j+1}}-\sqrt{\rho_{j}}}{\sqrt{\rho_{j+1}}+\sqrt{\rho_{j}}}
$$

(Szaraniec 1976). Such instantaneous reflection coefficients may be obtained using any resistivity profile. Since they are usually intended for use in relation to discontinuous media their application to smooth resistivity models is in the context of mapping the lateral continuity of gradients and turning points. Obviously, large reflection coefficients should be associated with major interface-like behaviour while zero values are obtained at turning points. To illustrate their characteristics in relation to a profile containing interfaces, a simplified model was constructed from the four- layer inversion result at site 8 . The model consists of layer 1 (resistivity $=120 \mathrm{ohm} . \mathrm{m}$, thickness $=1200 \mathrm{~m}$ ), layer 2 (resistivity $=15 \mathrm{ohm} \cdot \mathrm{m}$, thickness $=1300 \mathrm{~m}$ ), layer 3 (resistivity $=1 \mathrm{ohm} \cdot \mathrm{m}$, thickness $=1000$ $\mathrm{m}$ ) underlain by basement (a half-space of resistivity $=50 \mathrm{ohm} . \mathrm{m}$ ). The response of this model was 
generated across a frequency range from $100 \mathrm{~Hz}$ to $0.001 \mathrm{~Hz}$ and nominal $2 \%$ error estimates were assigned. A smooth OCCAM inversion of the synthetic data produced the resistivity profile shown in Fig. 9a, to an r.m.s. misfit of unity. A natural logarithm depth-scale is used and the horizontal lines denote the original (layered) model interface depths.

The broad characteristics of the original model can be seen to be represented by the behaviour of the smooth inversion, particularly in the changes in gradient that occur. The interpretational difficulty is that interface-behaviour has not been permitted. The reflection coefficient series obtained from the smooth profile is shown in Fig. 9b using an arbitrary gain factor. As can be seen in Fig. 9, the reflection coefficients, by forming a vertical derivative of the smooth profile, provide an 'amplification' of its behaviour. In Fig. 9b, the initial low-amplitude oscillation that occurs through layer 1 is false and due to slightly overfitting the data. Negative values indicate transitions to more conductive sequences. The first negative maximum clearly marks the base of layer 1 and the interface between layers 2 and 3 is represented by the largest negative maximum. A zero crossing occurs through the most conductive layer ( 3 ) since a positive excursion is required due to the transition to the less conductive basement.

Figure 9 provides an example of the behavioural characteristics of both a smooth solution and the reflection coefficient transformation when applied to a profile containing only interface behaviour. In the present application it has been stressed that the actual in situ behaviour is unlikely to be this simple, except in relation to the base of the basalt layer. In the present case, valid interpretations of the reflection coefficient transformation are made possible due to the well log control.

An example of the reflection coefficients determined from the smooth models at sites 8 and 10 is shown in Fig. 10, alongside the layered and smooth models. The reflection coefficients are determined here directly from the smooth solutions which are sampled uniformly in logarithmic depth at $\mathbf{3 0}$ layers per decade. Once again, negative values indicate transitions to more conductive sequences. In Fig. 10 the first maximum in the negative set of reflection coefficients is clearly associated with the base of the basalt (arrowed) while the deeper negative maximum indicates the maximum gradient in the decreasing resistivity profile. By cross-referencing the well-log information (Fig. 4) it can be seen that the reflection coefficients offer a different set of tie-in markers. The markers offered by the reflection coefficients consist of maximum and negative excursions together with zero crossings. In the case of the present data, the largest negative maximum is associated with the depth of the minimum resistivities found in the induction log (Fig. 4). These low resistivities, in turn, are associated with the conductive sand, siltstones and shales of the Irati, Palermo and Rio Bonito Formations.

The complete set of reflection coefficients across profile 5 is contoured in Fig. $8 \mathrm{c}$ for comparison with the previous two cross-sections. Although the amplitudes across the section could be equalized to investigate purely depth-dependent behaviour, by retaining 'true' amplitudes the section also indicates the true amplitude resistivity behaviour of Fig. 8a. This final section can be seen to combine the information contained in the previous two cross-sections in a formal way. Since the reflection coefficients derive from smooth, regularized inverse models we have found the procedure to be stable and in-formative when applied to the present (and other) data. As with the shadowrelief technique, the reflectivity transformation offers a useful mapping of the lateral correlation of the broad structural features across the basin. 


\section{Conclusions}

In this study the practical applicability of the MT technique to basin evaluation has been discussed. The specific case considered is an ongoing exploration problem in which $1200 \mathrm{~m}$ of basalt overlies Palaeozoic formations which contain source rocks with known hydrocarbon potential.

The study is limited, by data availability, to a quasi 1D interpretation of vertical structure. The value of the study lies in the demonstration of the practical limitations to the resolution of the geoelectric units using the control provided by a deep exploration well. Using models developed from the MT data it is stressed that the incorporation of the available information is a non-trivial exercise. A complicated relationship inevitably exists between the units resolved by the MT data and information obtained at the different scale of well logs.

The data detect three main units prior to electrical basement. The resistivity and thickness of the basalt cover are both highly resolved parameters. The complete Palaeozoic sedimentary sequence is represented by two units with the lower being expressed as a highly conducting (about 1 ohm.m) unit in the vicinity of formations which contain hydrocarbon source shales. At these depths (about $2500 \mathrm{~m}$ ) an offset occurs between the absolute resistivity, as determined by the surface soundings, and that returned by the induction log. The effect is more widespread than the survey discussed here; it persists in both 10 and 20 treatments of the data, and its significance is still being assessed.

Resistivity cross-sections across the basin have been constructed using both layered and smooth (non-interface) inversion results. The smooth, minimum-structure models offer a number of advantages over a conventional layered model construction scheme. In smooth model construction the number of interfaces need not be pre-defined and the resulting models appear more stable and less susceptible to parameter-equivalencing when cross-sections are assembled. Although obviously case- specific, it has also been suggested that at wave numbers appropriate to the resolution of the MT data, gradational (smooth) behaviour may be a more appropriate description of the in situ resistivity variation.

A regularized resistivity cross-section either developed, as here, from a set of smooth 1D models or from a full 2D inversion (deGroot-Hedlin and Constable 1990) will possess desirable stable attributes but can be difficult to interpret in conventional terms. It is suggested that techniques which enhance the intrinsic characteristics of smooth models can further reveal interface-type behaviour and permit a straightforward interpretation in terms of their lateral correlation. The application of a shadow relief technique and a reflectivity transformation have been demonstrated and their application to basin evaluation has been discussed using the control provided by a deep exploration well.

\section{Acknowledgements}

We thank Dr F. Nepomuenco of Petrobras Research Center for supporting this work. We also thank Gaston Fischer, Steve Constable and Catherine deGroot-Hedlin for the algorithm codes used in the inverse model assessments of the Clevelandia data. This paper is published with the approval of the Director, British Geological Survey (NERC).

\section{References}

BEAMISH, D. and TRAVASSOS, J .M. 1992. A study of static shift removal from magnetotelluric data. Journal of Applied Geophysics 29, in press. 
CONSTABLE, S.C., PARKER, R.L. and CONSTABLE, C .G. 1987. Occam's inversion: a practical algorithm for generating smooth models from electromagnetic sounding data. Geophysics 52, 289-300.

CHRISTOPHERSON, K .R. 1991. Applications of magnetotellurics to petroleum exploration in Papua New Guinea: a model for frontier areas. The Leading Edge, April 1991, 21-27.

DEGROOT-HEDLIN, C. and CONSTABLE, C. 1990. Occam's inversion to generate smooth, twodimensional models from magnetotelluric data. Geophysics 55, 1613-1624.

FISCHER, G. and LE QUANG, B.V. 1981. Topography and minimisation of the standard deviation in one-dimensional magnetotelluric modelling. Geophysical Journal of the Royal Astronomical Society 61,279-292.

GIBERT, D. and VIRIEUX, J. 1991. Electromagnetic imaging and simulated annealing, Journal of Geophysical Research 96, 8057-8067.

JIRACEK, G.R. 1990. Near-surface and topographic distortions in electromagnetic induction. Surveys in Geophysics 11, 163-203.

KELLER, G.V. 1987. Conductance map of the United States (progress report), Physics of the Earth and Planetary Interiors 41,216-225.

MILANI, E.J., KINOSHITA, E.M., ARAOJO, L.M. and da Cruz CUNHA, P.R. 1990. Parana Basin: petroleum potential in the depocenter area. Boletim de Geociencias da Petrobras 4, 21-34.

OLDENBURG, D. 1990. Inversion of electromagnetic data: an overview of new techniques. Surveys in Geophysics 11, 231-270.

ORANGE, A.S. 1989. Magnetotelluric exploration for hydrocarbons. Proceedings of the IEEE $11,287-$ 317.

PEDERSEN, J. and HERMANCE, J.F. 1986. Least-squares inversion of one-dimensional magnetotelluric data: an assessment of procedures employed by Brown University. Surveys in Geophysics 8, 187231.

PILKINGTON, M. and TODOESCHUK J.P. 1991. Naturally smooth inversions with a priori information from well logs. Geophysics 56, 1811-1818.

SMITH, J.T. and BOOKER, J .R. 1988. Magnetotelluric inversion for minimum structure. Geophysics $53,1565-1576$.

STANLEY, W.D., SAAD, A.R. and OHOFUGI, W. 1985. Regional magnetotelluric surveys in hydrocarbon exploration, Parana Basin, Brazil. Bulletin of the AAPG, 69, 346-360.

SZARANIEC, E. 1976. Fundamental functions for horizontally stratified earth. Geophysical Prospecting 24, 528-548.

ZALAN, P.V., CONCEICAO, J.C.J., WOLFF, S., ASTOLFI, M.A.M., VIEIRA, V.T., SANTOS NETO, E.V., CERQUEIRA, J.R., ZANOTO, O.A. and PAUMER, M.L. 1986. Analise de Bacia da Parana. GT- Os-009/85, PETROBRASIDEPEX, Rio de Janeiro. 


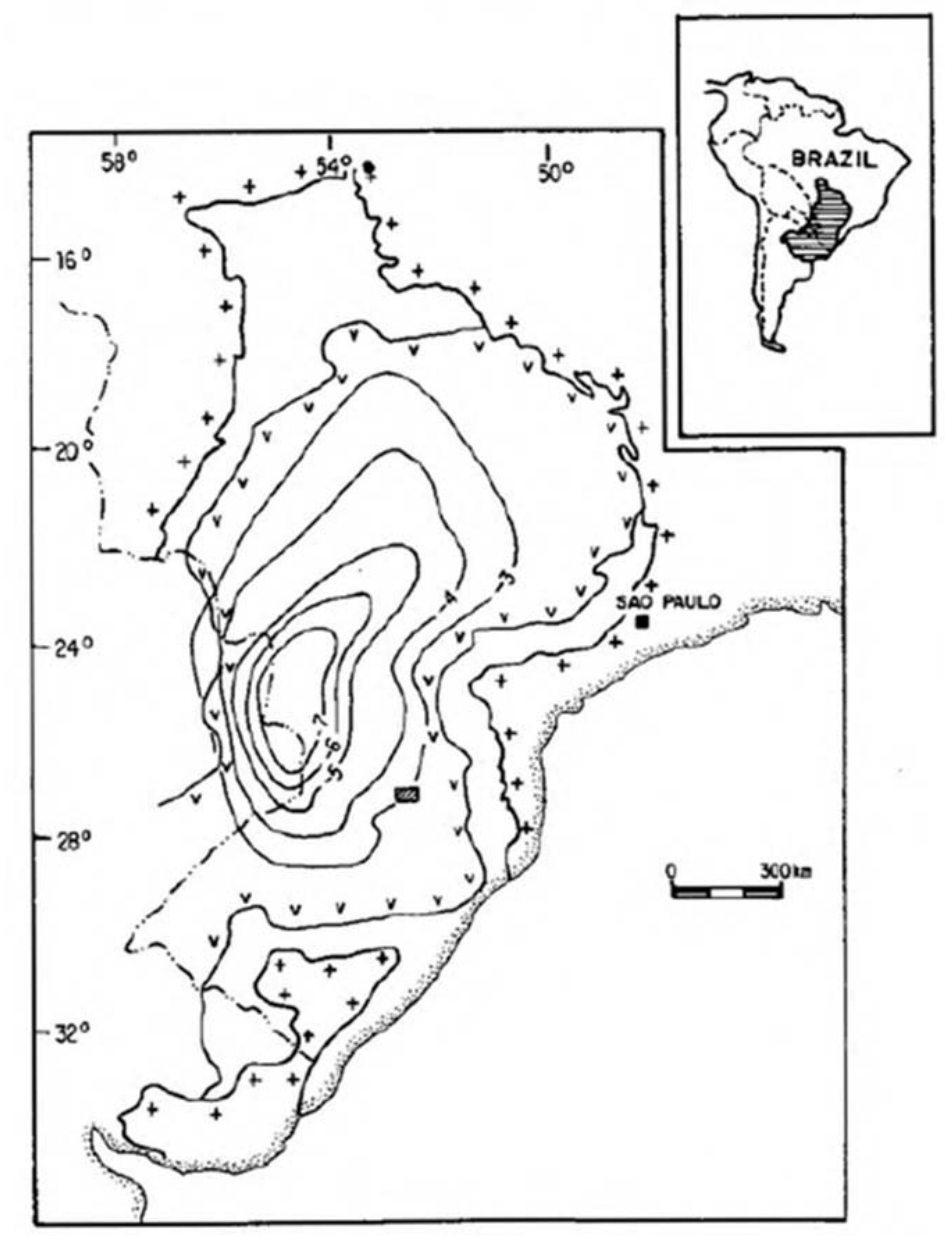

Fig. 1. Survey location map. Inset is Parana Basin shaded on map of South America. Main map shows extent of basalt cover ( $\mathrm{v}$ symbols) and structural contours of depth to seismic basement in $\mathrm{km}$ (after Zalan et al. 1986). Contour interval is $1 \mathrm{~km}$. Position of Clevelandia MT array is shown by the crosshatched rectangle (not to scale). 


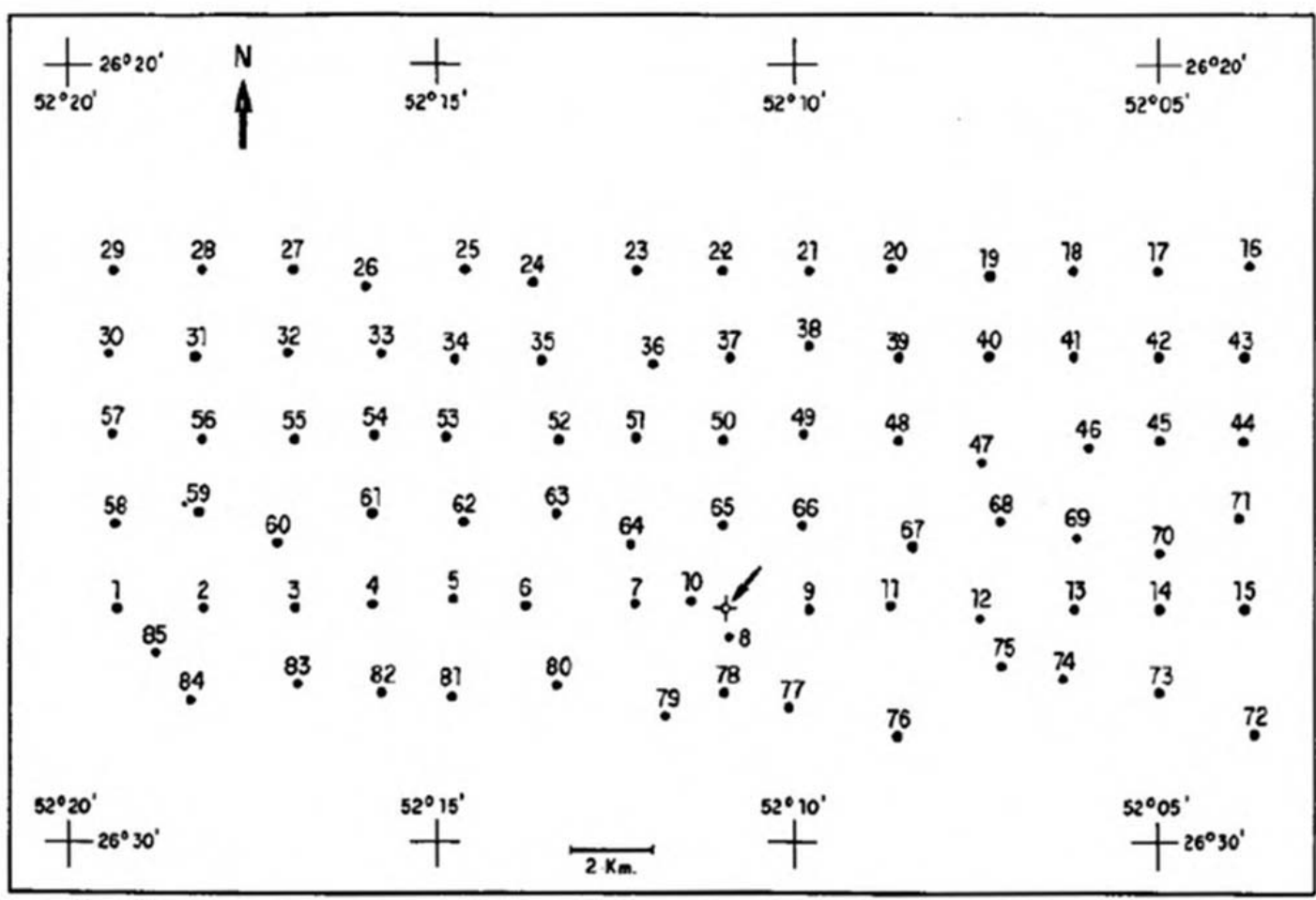

Fig. 2. Sounding locations of the MT grid array and exploration well 2-AL-1-SC (arrowed). E-W 'profile 5 ' consists of locations 1 through to 15 


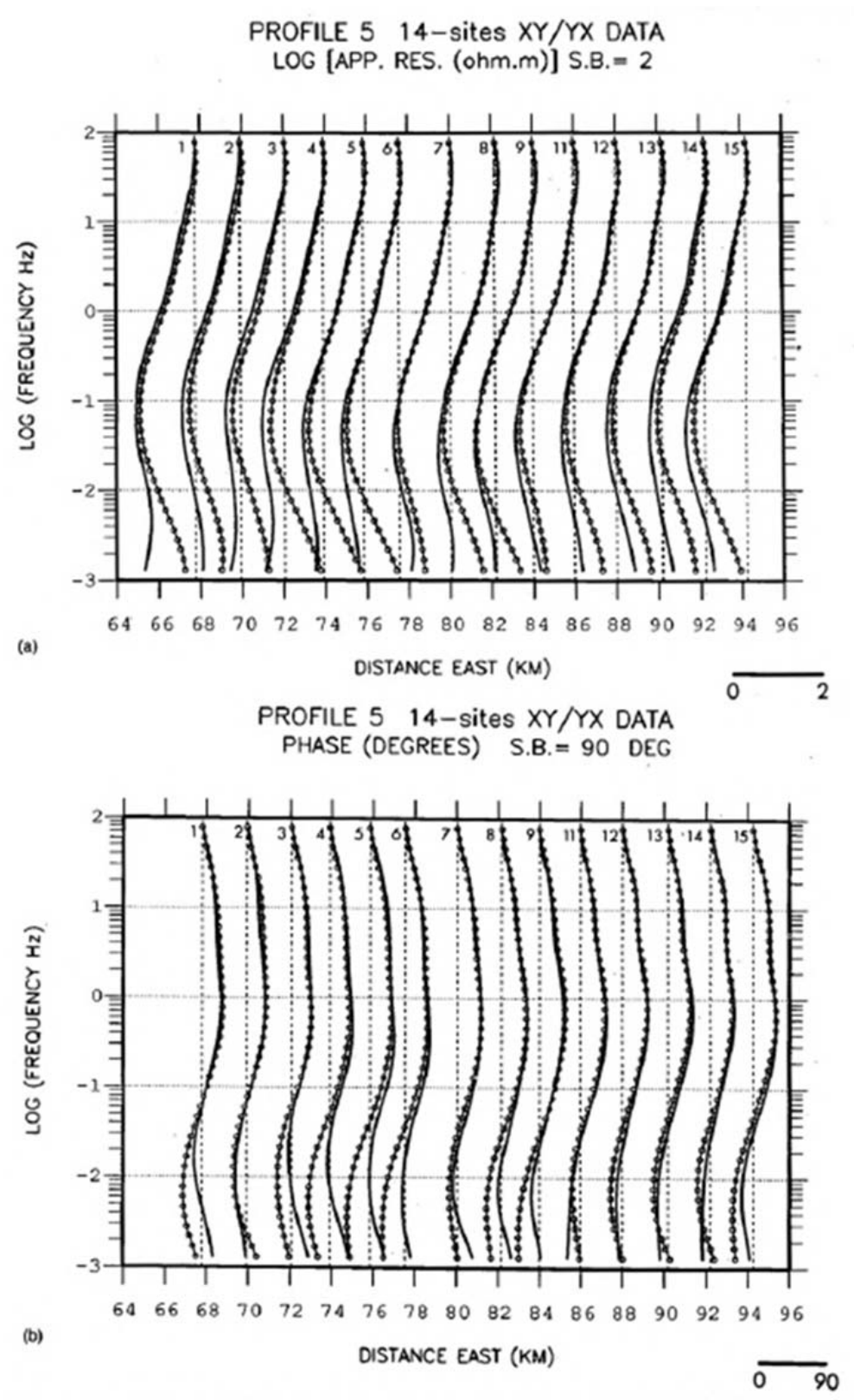

Fig. 3. Corrected sounding data across profile 5 with site 10 omitted for clarity. Data plotted as a function of easting location, indicated by vertical dash lines. xy component shown as line with symbols, yx component is line only. (a) Apparent resistivity data in logarithmic scale, scale bar (bottom right) represents two decades. (b) Phase data in linear scale, scale bar (bottom right) represents $90^{\circ}$. 


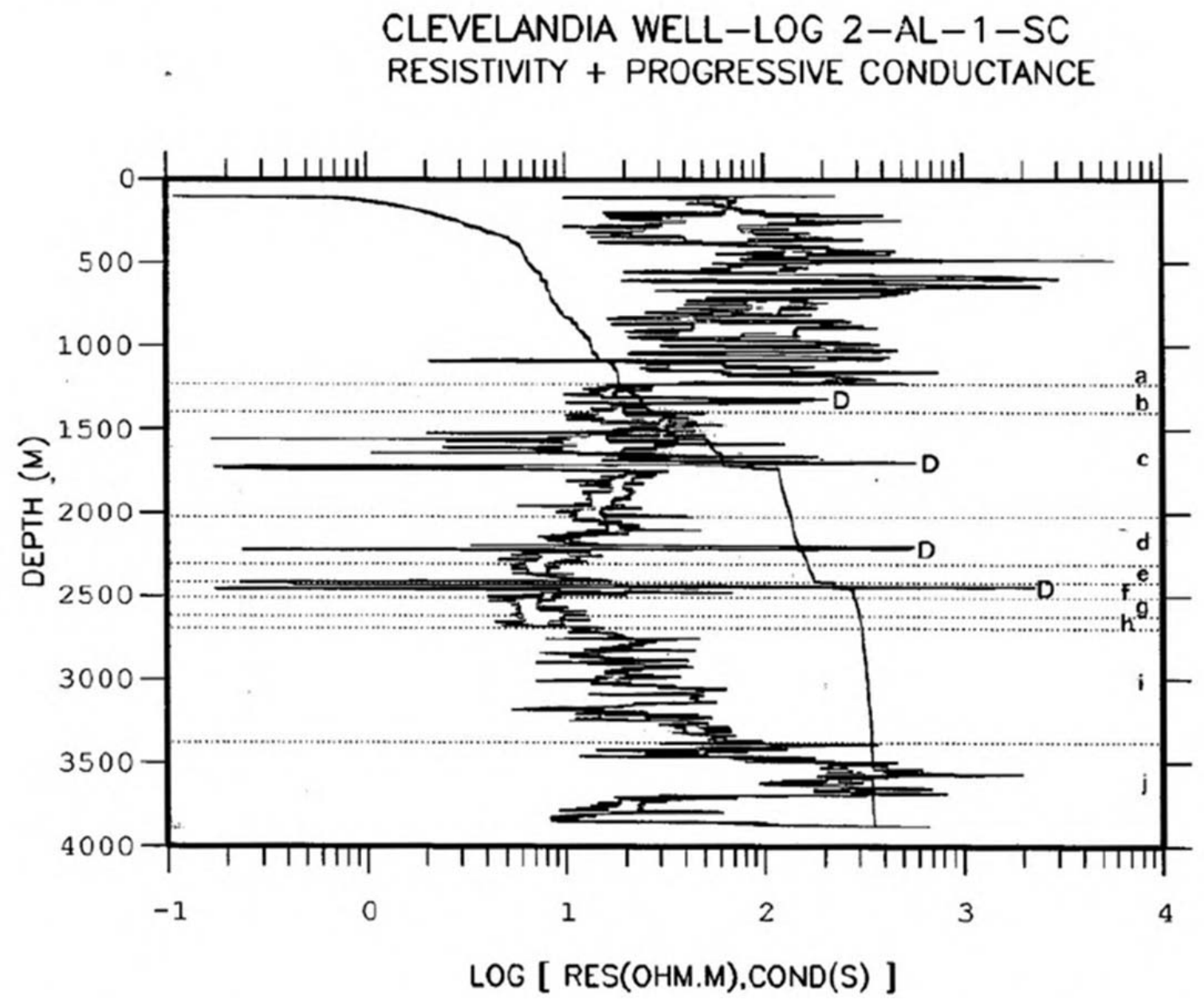

Fig. 4. Induction log, progressive conductance and stratigraphy from well 2-AL-1-SC. Resistivity (ohm.m) and conductance (S) shown on the same logarithmic scale. Stratigraphy denoted by horizontal dotted lines and lower case letters. Formations denoted are $a=$ Serra Geral basalt, $b=$ Botucato, $\mathrm{c}=$ Rio do Rasto, $\mathrm{d}=$ = Terezina, $\mathrm{e}=$ Serra Alta $, \mathrm{f}=\operatorname{Irati}, \mathrm{g}=$ Palermo, $\mathrm{h}=$ Rio Bonito, $\mathrm{i}=$ Harare and $\mathrm{j}=$ Furnas Formation. $\mathrm{D}$ denotes diabase sill. 

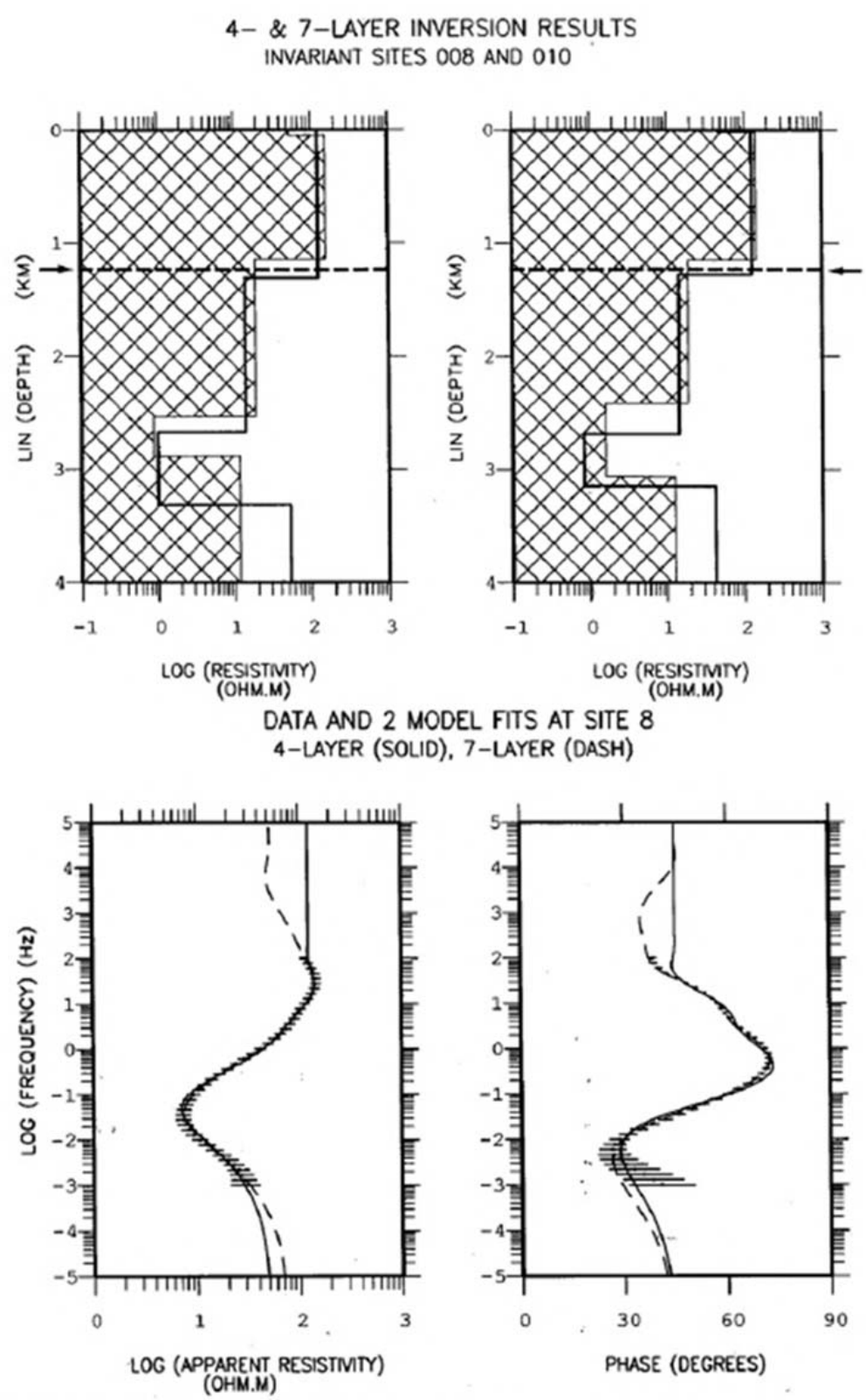

Fig. 5. (a) Layered inversion results at sites 8 (left) and 10 (right). Four-layer result is single line and seven-layer result is shaded. Base of the basalt is horizontal dash line (arrowed). (b) Response of the two models at site 8 together with observed response. Observed data shown with error bars, fourlayer model response is solid line, seven-layer model response is dash line. 


\section{4-LAYER AND SMOOTH MODELS \\ PROFILE 514 SITES}

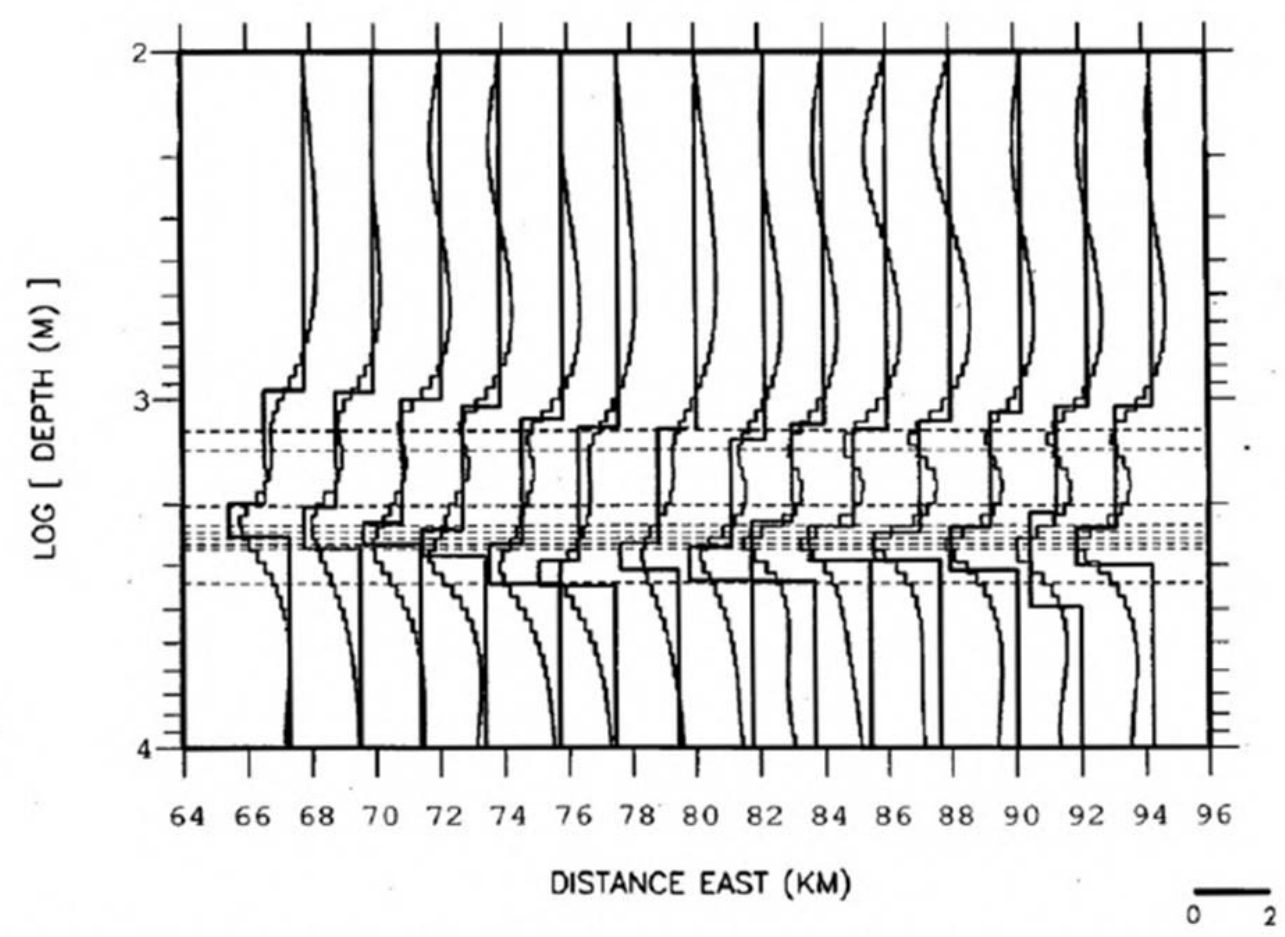

Fig. 6. Four-layer and smooth inversion results across profile 5 with site 10 omitted for clarity. Logarithmic depth and resistivity scales. Results plotted as a function of easting location. Scale bar (bottom right) represents 2 decades. Horizontal dash lines are stratigraphic markers, as in Fig. 4. 

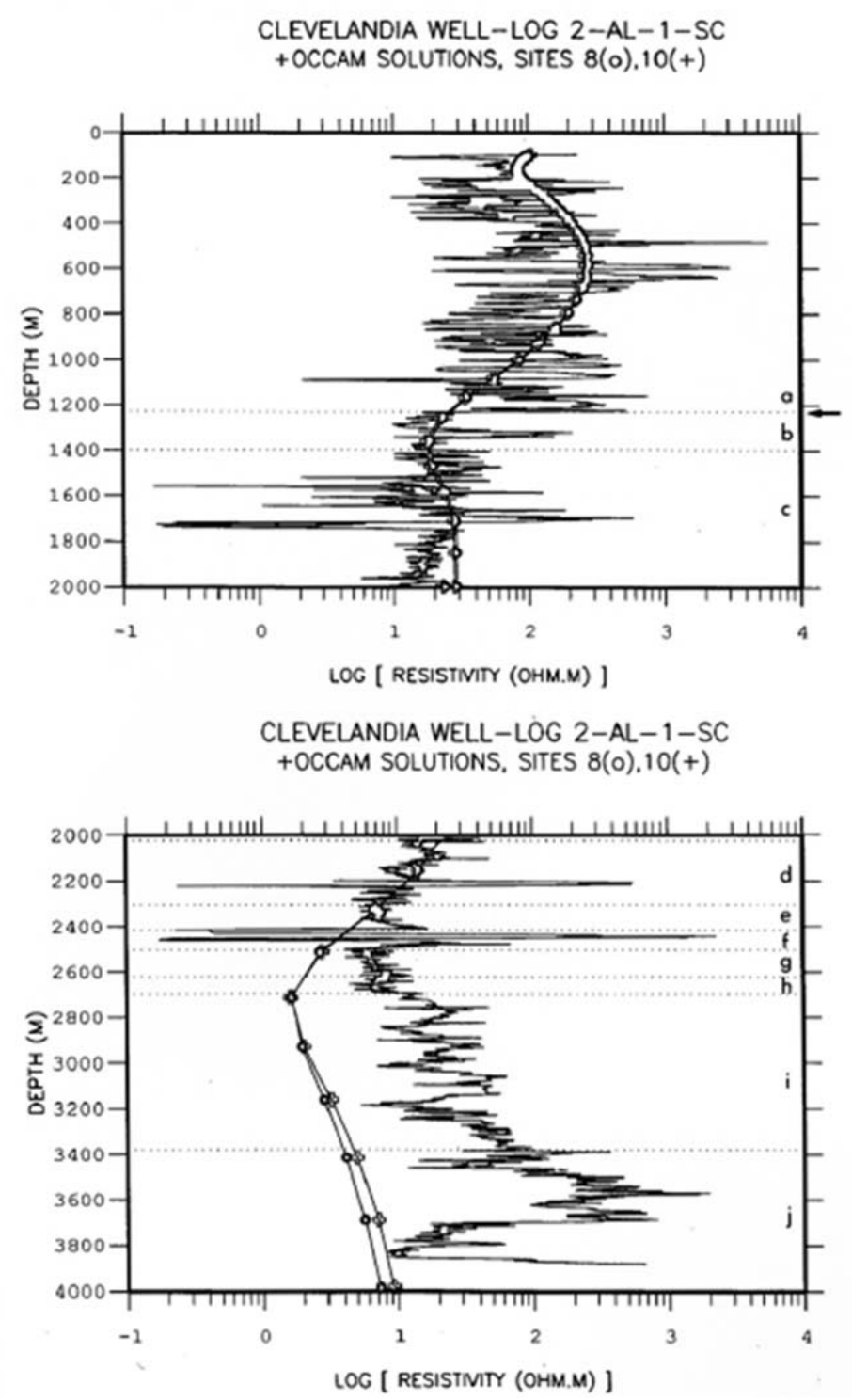

Fig. 7. Observed induction log compared with smooth inversion models at sites 8 (solid circles) and 10 (open crosses). Stratigraphy denoted by horizontal dotted lines and lower case letters as in Fig. 4. Depth ranges 0-2000 m (upper) and 2000-4000 m (lower). 
TRUE SCALE

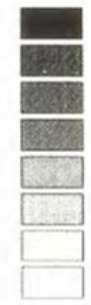

a)

(b)

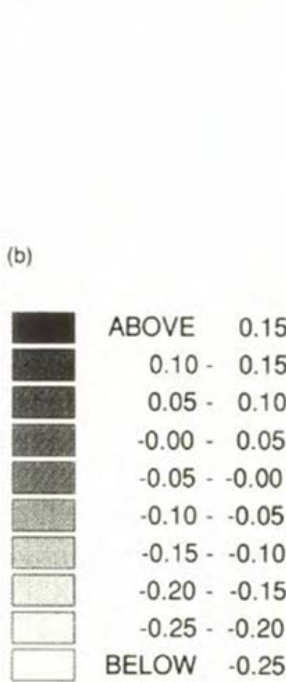

BELOW 0.50

(c)

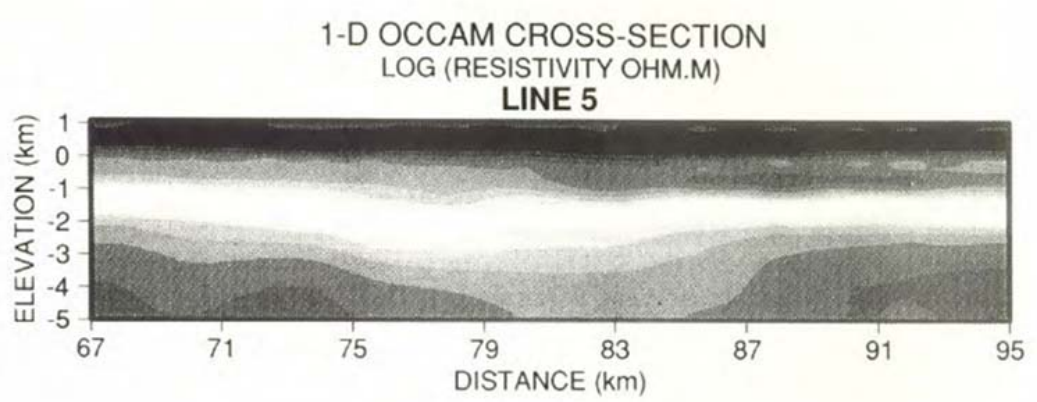

SHADOW-RELIEF
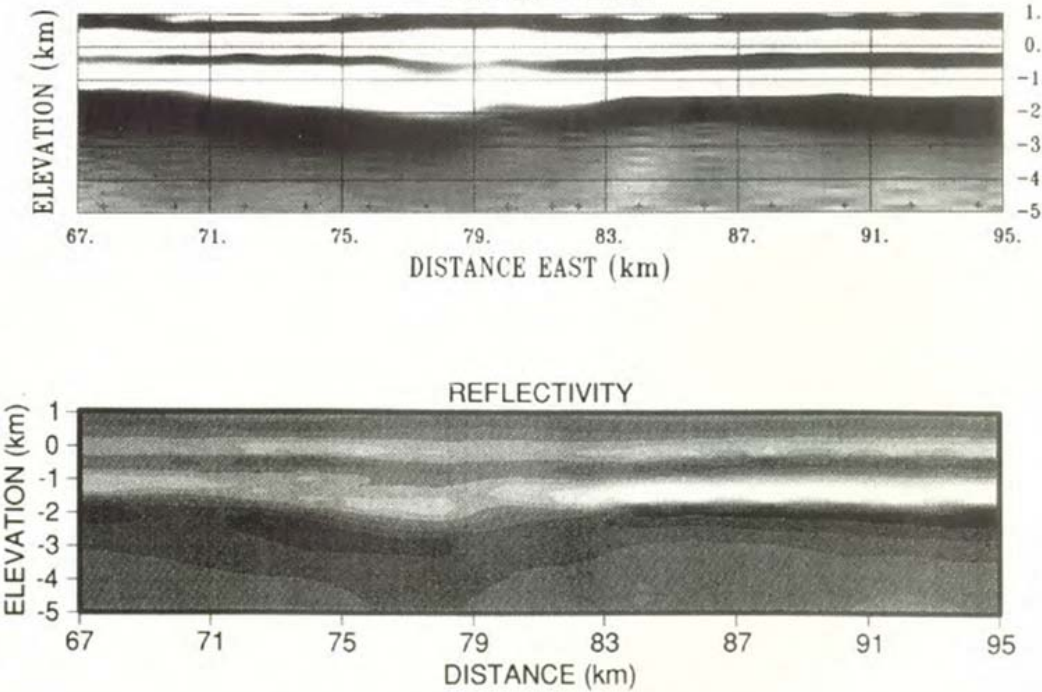

Fig. 8. Three cross-sections across profile 5. Corrected for site elevations. Site eastings (1-15) indicated by cross marks in (b). (a) Resistivity (ohm.m) on a logarithmic scale. Contour interval 0.25 ohm.m. (b) Resistivity cross-section processed using shadow relief. Light source elevation 45 degrees, azimuth 180 degrees. No scale. (c) Reflectivity obtained from resistivity cross-section. True amplitudes at a contour interval of 0.05 . 


\section{1-D smooth OCCAM solution \\ synthetic $r \mathrm{~ms}=1.0$}
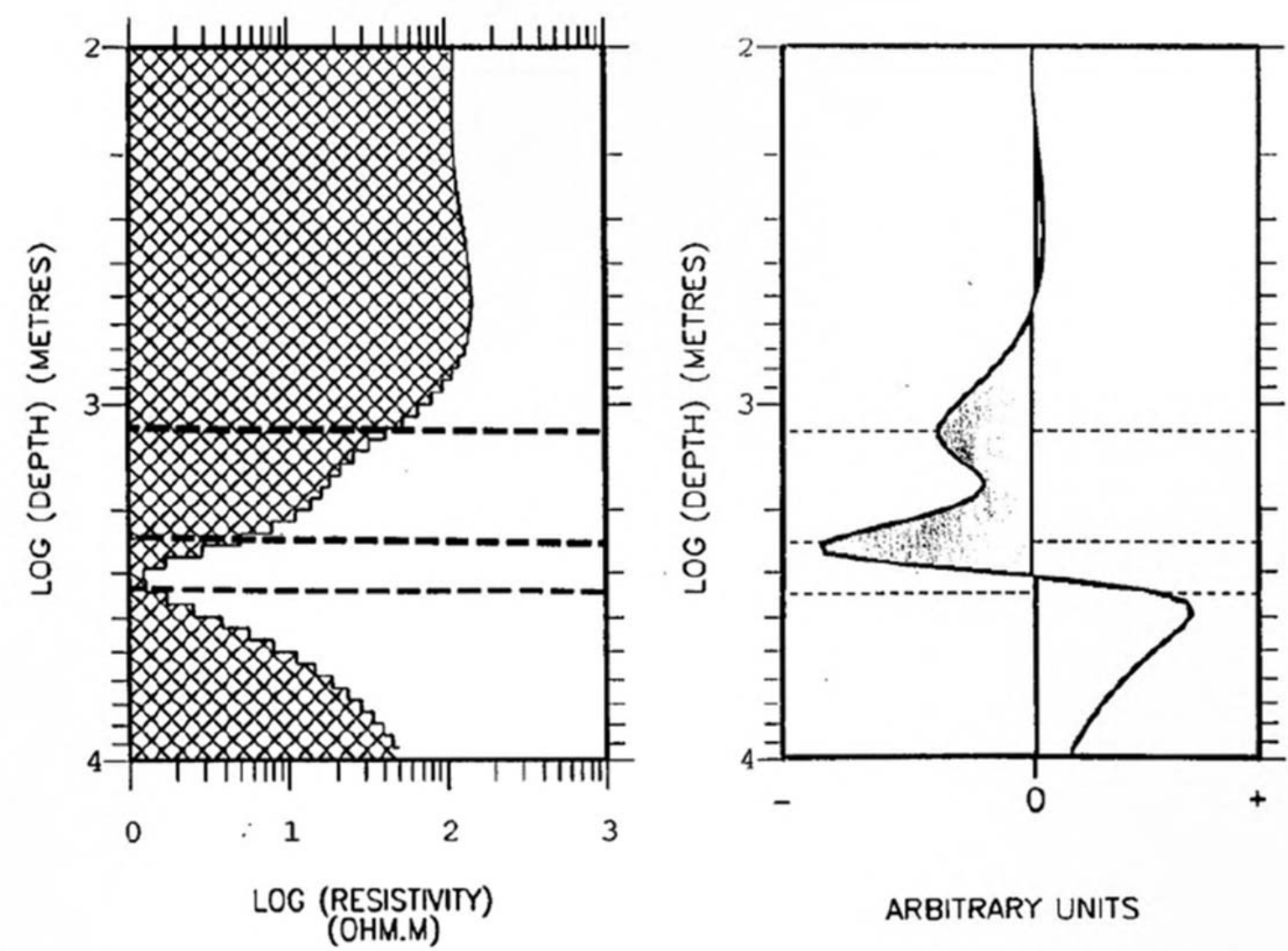

Fig. 9. Left: OCCAM inversion model obtained from data generated from four-layer synthetic model. Right: Reflectivity transformation obtained from the OCCAM model. Horizontal dash lines indicate the interfaces of the synthetic model described in the text. 
COMPARISON OF MODELS AT SITES 8,10

LAYERED, SMOOTH AND REFLECTIVITY

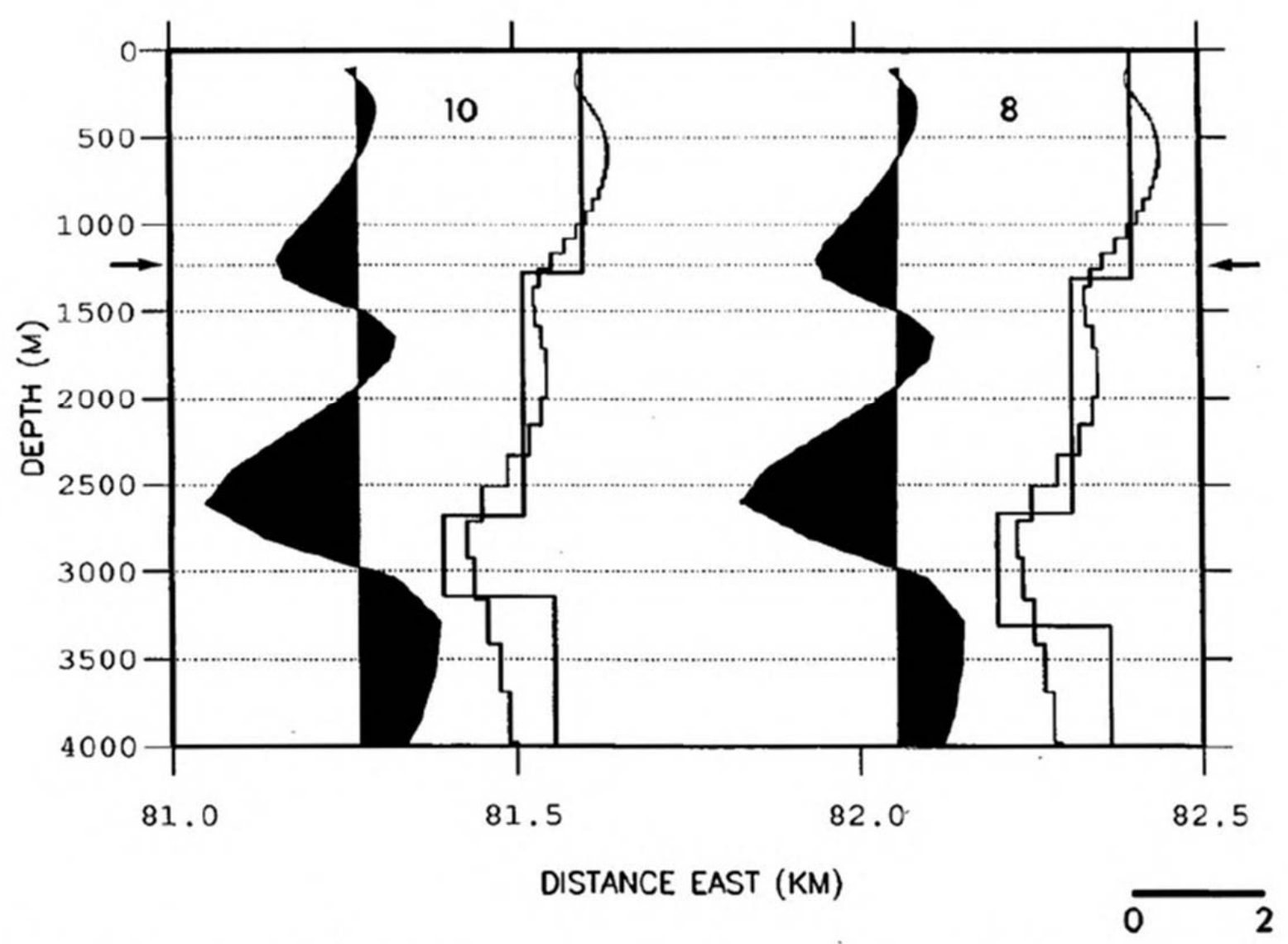

Fig 10. Comparison of four-layered and smooth inversion results and reflectivity transformation at sites 10 (left) and 8 (right). Horizontal dash lines are for reference except for arrow at $1230 \mathrm{~m}$ which denotes base of the basalt. Resistivity models use logarithmic scale and scale bar (2 decades) is shown at bottom right. 